\title{
Rules for Aggregating Information
}

\author{
Christopher P. Chambers* and Alan D. Miller ${ }^{\dagger \ddagger}$
}

May 1, 2010

\begin{abstract}
We present a model of information aggregation in which agents' information is represented through partitions over states of the world. We discuss three axioms, meet separability, upper unanimity, and non-imposition, and show that these three axioms characterize the class of oligarchic rules, which combine all of the information held by a pre-specified set of individuals.

JEL classification: D70, D71, D72
\end{abstract}

\section{Introduction}

The difficulty of appropriately defining rules for sharing information in differential informa-

tion economies is well-documented. Efficiency and core concepts are necessarily information dependent. Privacy of information opens the door to strategic manipulation of information

${ }^{*}$ Division of the Humanities and Social Sciences, Mail Code 228-77, California Institute of Technology, Pasadena, CA 91125. Email: chambers@caltech.edu Web: http://www.hss.caltech.edu/ chambers/

${ }^{\dagger}$ Faculty of Law and Department of Economics, University of Haifa, Mount Carmel, Haifa, 31905, Israel. Email: admiller@econ.haifa.ac.il Web: http://econ.haifa.ac.il/ admiller/

${ }^{\ddagger}$ The authors would like to thank the editors, two anonymous referees, as well as Elad Dokow, Federico Echenique, Debasis Mishra, Ariel Rubinstein, Dov Samet, and Eran Shmaya for their comments. Christopher P. Chambers gratefully acknowledges support by the National Science Foundation, SES-0751980. 
revelation on the part of agents. Early works on efficiency concepts in differential information economies include Wilson (1978) and Holmstrom and Myerson (1983).

Here we point out a different difficulty with information sharing. Imagine two agents, each of whom face two distinct signals about the true state of the world. Agent 1 observes whether or not the sun comes out, and Agent 2 observes whether or not the temperature falls below freezing. These are "weather" signals. Likewise, Agent 1 observes the demand for oranges in New York, whereas Agent 2 observes the demand for oranges in New England. These are "demand" signals. The two agents agree ahead of time on a prespecified rule that is to be used in aggregating signals, resulting in a group signal. The rule need not recommend sharing of all information between the agents. The agents can apply their rule to the weather signals; likewise they can apply their rule to the demand signals. The resulting aggregates are a group weather signal and a group demand signal.

On the other hand, each agent has a large signal about "weather and demand," which results from combining her private signal about weather with her private signal about demand. This agent uses this combined "weather and demand" signal to predict the price of orange futures. When aggregating the "weather and demand" signals across agents, the rule produces a group "weather and demand" signal. In general, it need not be the case that the group "weather and demand" signal is the same signal that results from combining the group weather and group demand signal. This fact opens the door to strategic manipulation of the rule - how signals are specified becomes relevant for determining the final group signal. In order to rule out the possibility of such manipulation, we require that these group signals always coincide: a property we call meet separability.

Formally, all informational content of a signal in our model is captured by a partition 
of the states of the world. Therefore, a signal is identified with a partition over states of the world.

We consider two other requirements that a rule should satisfy. The first, upper unanimity, says that when all agents face an identical signal, the group signal should be a coarsening of this one. (In general, we might require that the group signal is a coarsening of the coarsest common refinement; this turns out to be implied by our axioms.) The second, non-imposition, simply states that the rule is non-imposed; that is, any conceivable signal might be the realized signal of the group for some profile of individual signals.

These three axioms, taken together, characterize the oligarchic rules. A rule is oligarchic if there is a prespecified group of agents (the oligarchy) who determine the outcome of the rule. In our case, we take the group signal of an oligarchic rule to be the totality of information contained in the signals of the oligarchy (the coarsest common refinement of their signals). Our main result states that a rule satisfies meet separability, upper unanimity, and non-imposition if and only if it is oligarchic. Together with a basic symmetry axiom, this states that the only information sharing rule satisfying the three properties is the rule which always aggregates all information.

\subsection{Related Literature}

The oldest result on the aggregation of partitions is an Arrovian-style impossibility theorem discovered by Mirkin (1975), and refined by Leclerc (1984). ${ }^{1}$ This theorem characterized oligarchic rules as the class of rules satisfying an Arrovian-style independence condition

\footnotetext{
${ }^{1}$ See also Barthélemy et al. (1986), Fishburn and Rubinstein (1986), Barthélemy (1988), and Day and McMorris (2003). More recently, Nehring (2006) provides a proof of Mirkin's theorem using tools created to study judgment aggregation.
} 
and a unanimity condition. The independence axiom requires that individual signals about demand in New England be ignored when determining the collective signal about demand in New York. The unanimity condition applies when each individual receives the same signal. In that case, the commonly-received signal becomes the collective signal.

Miller (2008) investigates the concept of meet separability in the context of group identification. Vannucci (2008) studies the property in a more general model of group identification in which opinions may be in the form of partitions of the society.

Recently, others have independently proven a result similar to our own. Dimitrov et al. (2009) characterize oligarchic rules as the class of rules satisfying meet separability and two other axioms, pareto-plus and non-triviality. Pareto-plus requires that, if none of the individual signals distinguish between two states ("rain" and "clouds"), then the group signal also does not distinguish between those two states. Pareto-plus implies the upper unanimity axiom. The non-triviality axiom requires that the group signal must be informative for some profile of individual signals. Non-triviality is implied by the non-imposition axiom. The proof of their theorem, however, is completely different from our own.

\section{The Model}

\subsection{Notation and the Model}

The set of agents is a finite set $N \equiv\{1, \ldots, n\}$ of individuals. States of the world are elements of a finite set $\Omega$. A signal is a partition over $\Omega$. Formally, a signal can be identified with an equivalence relation $p \subseteq \Omega \times \Omega$. Two states stand in the relation if they cannot be distinguished according to the signal. 
Let $\mathscr{E}$ denote the set of equivalence relations on $\Omega$. Let $\mathscr{P}$ denote the set of partitions of $\Omega$. There is a one to one correspondence between $\mathscr{E}$ and $\mathscr{P}$ such that two states $\omega$ and $\omega^{\prime}$ are equivalent if and only if they are in the same cell of the partition. While this paper focuses on equivalence relations, all results apply to partitions. ${ }^{2}$

The set $\mathscr{E}$ forms a lattice when ordered under set inclusion. A profile is an $N$ dimensional vector of equivalence relations $P \equiv\left(P_{1}, \ldots, P_{n}\right) \in \mathscr{E}^{N}$, one for each agent. The set $\mathscr{E}^{N}$ also forms a lattice under the product order. For any two elements $P$ and $Q$ of a lattice, we denote by $P \wedge Q$ their greatest lower bound, and by $P \vee Q$ their least upper bound. An aggregator is a function $f: \mathscr{E}^{N} \rightarrow \mathscr{E}$ mapping from signal profiles into the aggregate signal.

\subsection{The axioms and the main result}

Our first axiom eliminates strategic manipulation by framing of signals. It was described at length in the introduction.

Meet Separability: For all $P, Q \in \mathscr{E}^{N}, f(P) \wedge f(Q)=f(P \wedge Q)$.

Our next axiom is a very weak unanimity property.

Upper Unanimity: If $P_{i}=P_{j}$ for all $i, j \in N$, then $f(P) \geq P_{1}=\ldots=P_{n}$.

Lastly, we require that, for any signal, there is a signal profile realizing that signal.

Non-Imposition: For all $p \in \mathscr{E}$, there exists an $Q \in \mathscr{E}^{N}$ such that $f(Q)=p$.

\footnotetext{
${ }^{2}$ For information on lattices and equivalence relations, please see Blyth and Janowitz (1972); Birkhoff (1973); Aigner (1979); Szasz (1964); Ore (1942); Davey and Priestley (2002); Grätzer (2003); Roman (2008). We thank the associate editor and an anonymous referee for these references.
} 
The next definition defines a class of rules. An oligarchy is a maximal set of agents whose signals are combined to make the group signal.

Oligarchic Aggregator: There exists $S \subseteq N, S \neq \varnothing$, such that $f(P) \equiv \bigwedge_{i \in S} P_{i}$.

Theorem 1. An aggregator satisfies meet separability, upper unanimity, and nonimposition if and only if it is oligarchic. Moreover, all three axioms are independent.

The proof of the theorem can be found in the appendix.

\subsection{Anonymity}

The principle of anonymity requires that each agent's signal be given the same weight. Formally, let individuals trade signals amongst themselves according to a permutation $\pi$ : $N \rightarrow N$ of the set of agents. Anonymity requires that the collective signal should not change because agents have traded their signals.

Anonymity: For all $\pi$ and $P \in \mathscr{E}, f(P)=f\left(P_{\pi(1)}, \ldots, P_{\pi(n)}\right)$.

The only oligarchic aggregator which satisfies anonymity is the one which includes all of the agents' information. The proof of the following proposition is trivial and is left for the readers.

Proposition 2. An aggregator $f$ satisfies meet separability, upper unanimity, nonimposition, and anonymity if and only if $f(P) \equiv \bigwedge_{i \in N} P_{i}$.

\section{Meet Homomorphisms and Inverse Functions}

To prove Theorem 1, we use a more general result about finite lattices. 
Let $A, Z$ be finite lattices. Without loss of generality we denote the orders corresponding to the lattices by $\leq$. Any finite lattice has a maximal and minimal element. Without loss of generality we denote these by $\mathbf{1}$ and $\mathbf{0}$ respectively.

We say that a function $f: A \rightarrow Z$ is a meet-homomorphism if for all $a, b \in A, f(a \wedge b)=$ $f(a) \wedge f(b)$. Likewise, a function is a join-homomorphism if for all $a, b \in A, f(a \vee b)=$ $f(a) \vee f(b)$.

A function $f: A \rightarrow Z$ is monotonic if for all $a, b \in A, a \leq b$ implies $f(a) \leq f(b)$. Defining $x<y$ to mean $x \leq y$ and $x \neq y$, say $f$ is strictly monotonic if for all $a, b \in A$, $a<b$ implies $f(a)<f(b)$. We say that $f$ is surjective if for all $z \in Z$, there exists $a \in A$ such that $f(a)=z$. We say that $f$ is injective if for all $a, b, f(a)=f(b)$ implies $a=b$.

Theorem 3. A function $f: A \rightarrow Z$ is a surjective meet-homomorphism if and only if there exists an injective join-homomorphism $g: Z \rightarrow A$ satisfying $g(\mathbf{0})=\mathbf{0}$ such that, for all $a \in A$, both $g(f(a)) \leq a$ and

$$
f(a)=\bigvee\{z \in Z: g(z) \leq a\}
$$

The proof of the theorem can be found in the appendix.

\section{Conclusion}

We have presented a model of information aggregation and have characterized oligarchic aggregation rules as the unique class of rules satisfying meet separability, upper unanimity, and non-imposition. Oligarchic aggregation rules combine all of the information received by a pre-selected set of agents. Other rules will necessarily violate one of the three axioms. Rules 
which violate the meet separability axiom allow the possibility of strategic manipulationthe group signals may be affected by the questions asked. Independent questions about the weather and about demand for oranges may lead to different conclusions about the price of orange futures than would follow from a more direct question. Rules which violate upper unanimity and non-imposition have very undesirable properties - the resulting group signals are derived from something other than the agents' information.

Of interest for future research is the investigation of the aggregation of modal operators. Instead of working with partitions directly, one would work with the corresponding knowledge operators, and investigate axioms placed directly on these.

\section{Appendices}

\section{A Proofs}

We prove the theorems in reverse order.

\section{A.1 Proof of Theorem 3}

Proof. All references are to Blyth and Janowitz (1972); terminology can be found there as well..$^{3}$

First, because $f$ is surjective, we know that for all $z \in Z,\{a \in A: f(a) \geq z\} \neq \varnothing$. Because $f$ is a meet-homomorphism, it follows that $f$ is monotonic. Consequently, $f$ is quasi-residual. (See page 9.) Because $f$ is a quasi-residual meet homomorphism, it follows

\footnotetext{
${ }^{3}$ We thank an anonymous referee for showing us how this proof could be established from known results in the literature.
} 
from Theorem 5.2 that it is residual. Let $g: Z \rightarrow A$ be the residuated mapping having $f$ as its residual. Then, for all $a \in A, g(f(a)) \leq a$ and $f$ has the following representation (page 11):

$$
f(a)=\bigvee\{z \in Z: g(z) \leq a\}
$$

Because $f$ is surjective, it follows from Theorem 2.6 that $g$ is injective. Lastly, as $g$ is residuated, it follows from Theorem 5.2 that it is a quasi-residuated join-homomorphism. Therefore $\{z \in Z: g(z) \leq \mathbf{0}\} \neq \varnothing$. Because $g$ it is one-to-one and monotone; we conclude that $g(\mathbf{0})=\mathbf{0}$.

Conversely, suppose that $f$ has the representation as described in the theorem. Then $f$ is residual, and is the residual of $g$. (See page 11.) Because $f$ is residual, it follows from Theorem 5.2 that it is a meet homomorphism. Since $g$ is injective, Theorem 2.6 implies that $f$ must be surjective.

\section{A.2 Proof of Theorem 1}

Proof. Step 1. As noted in section 2.1, the set $\mathscr{E}$ forms a lattice under set inclusion, and the set $\mathscr{E}^{N}$ thus forms a lattice under the product order. The meet separability and nonimposition axioms imply that $f$ is a meet-homomorphism and is a surjection, respectively. As a consequence, Theorem 3 implies that there is an injective join-homomorphism $g: \mathscr{E} \rightarrow$ $\mathscr{E}^{N}$ satisfying $g(\mathbf{0})=\mathbf{0}$, such that, for all $P \in \mathscr{E}^{N}$, both $g(f(P)) \leq P$ and

$$
f(P)=\bigvee\{p \in \mathscr{E}: g(p) \leq P\}
$$


Let $J \equiv\{p \in \mathscr{E}: p>\mathbf{0}, p \geq r>\mathbf{0}$ implies that $p=r\}$ be the set of atoms of $\mathscr{E}$. For each atom there is a unique pair of states of the world $\alpha, \beta \in \Omega$ which are equivalent. The lattice of partitions is atomistic: every nonzero element of $p \in \mathscr{E}$ can be expressed as the finite join of elements of $J$. (See Roman, 2008, pp. 80, 110.)

Step 2. We show that there is a non-empty subset $S \subseteq N \backslash \varnothing$ such that, for all $p \in J$, $g(p)_{i}=p$ if $i \in S$, and $g(p)_{i}=\mathbf{0}$ if $i \notin S$.

Let $p \in J$. By upper unanimity, $f(p, \ldots, p) \geq p$. From step one, $g(f(p, \ldots, p)) \leq(p, \ldots, p)$. Therefore, from the monotonicity of $g$, it follows that $(p, \ldots, p) \geq g(f(p, \ldots, p)) \geq g(p)$. From step one, $g$ is injective and $g(\mathbf{0})=\mathbf{0}$. Therefore, because $p>\mathbf{0}$, it follows that $(p, \ldots, p) \geq g(p)>\mathbf{0}$. This implies that there is a subset $S \subseteq N \backslash \varnothing$ such that $g_{i}(p)=p$ if $i \in S$ and $g_{i}(p)=\mathbf{0}$ if $i \notin S$. If $|\Omega|<3$, we may proceed to the last step. Otherwise, define $S^{p} \equiv\left\{i \in N: g(p)_{i}=p\right\}$. Let $q \in J \backslash\{p\}$, and define $S^{q}$ accordingly. To complete this step we must show that $S^{p}=S^{q}$.

Let $r, t \in J \backslash\{p\}$ such that $r \vee t \geq p$ and define $S^{r}$ and $S^{t}$ accordingly. ${ }^{4}$ Because $g$ is a join-homomorphism, it follows that $g(r) \vee g(t)=g(r \vee t) \geq g(p)$. Now $(g(r) \vee g(t))_{i} \geq$ $g_{i}(p) \geq p$ if and only if $i \in S^{r} \cap S^{t}$, which implies that $S^{p} \subseteq S^{r} \cap S^{t}$. Next, because $r \vee t \geq p$ it follows that $p \vee r \geq t$ and $p \vee t \geq r$. By repeating this argument, it follows that $S^{r} \subseteq S^{p} \cap S^{t}$ and $S^{t} \subseteq S^{p} \cap S^{r}$. Therefore $S^{p}=S^{t}=S^{r}$.

If there exists $s \in J \backslash\{p, q\}$ such that $p \vee q \geq s$, this concludes the step. If not, we can always find $r, s, t \in J \backslash\{p, q\}$ such that $p \vee r \geq s$ and $q \vee r \geq t$ and therefore $S^{p}=S^{r}=S^{q} .^{5}$

\footnotetext{
${ }^{4}$ To see why $r$ and $t$ necessarily exist, recall that for each atom in $J$, there is a unique pair of states of the world which are equivalent. Without loss of generality, let the unique pair for $p$ be $\alpha, \beta \in \Omega$, and refer to $p$ by this pair, so that $\langle\alpha \beta\rangle \equiv p$. Because $|\Omega| \geq 3$, there must exist another element $\gamma \in \Omega \backslash\{\alpha, \beta\}$ and, consequently, distinct atoms $r \equiv\langle\alpha \gamma\rangle$ and $t \equiv\langle\beta \gamma\rangle$. In the partition denoted by $\langle\alpha \gamma\rangle \vee\langle\beta \gamma\rangle$, the pair $\alpha$ and $\gamma$ are equivalent and the pair $\beta$ and $\gamma$ are equivalent. It follows that $\alpha$ and $\beta$ must also be equivalent, so therefore $\langle\alpha \gamma\rangle \vee\langle\beta \gamma\rangle \geq\langle\alpha \beta\rangle$.

${ }^{5}$ To see why $r, s, t$ necessarily exist in this case, suppose that $p=\langle\alpha \beta\rangle$ and $q=\langle\alpha \gamma\rangle$. Then $p \vee q=$
} 
Step 3. We show that there is a non-empty subset $S \subseteq N \backslash \varnothing$ such that, for all $p \in \mathscr{E}$, $g_{i}(p)=p$ if $i \in S$, and $g_{i}(p)=\mathbf{0}$ if $i \notin S$.

We have shown that there exists $S \subseteq N$ such that, for all $q \in J, g_{i}(q)=q$ if $i \in S$ and $g_{i}(q)=\mathbf{0}$ if $i \notin S$. Let $P \in \mathscr{E}^{N}$. The result now follows as every nonzero element $p \in \mathscr{E}$ can be expressed as a finite join of elements of $J$, and as $g$ is a join homomorphism.

Step 4. Completing the proof.

Recall $f(P)=\bigvee\{p \in \mathscr{E}: g(p) \leq P\}$. The statement $g(p) \leq P$ is true if and only if for all $i \in S, p \leq P_{i}$, which is true if and only if $p \leq \bigwedge_{i \in S} P_{i}$. Consequently, $f(P)=$ $\bigvee\left\{p \in \mathscr{E}: p \leq \bigwedge_{i \in S} P_{i}\right\}=\bigwedge_{i \in S} P_{i}$

Independence of the Axioms. We present three rules; each satisfies two of the axioms but violates the third.

Rule 1. The degenerate rule, $f(P)=\mathbf{1}$ for all $P \in \mathscr{E}^{N}$. This rule trivially satisfies meet separability and upper unanimity but violates non-imposition.

Rule 2. The rule $f(P)=P_{2}$, if $P_{1}=\mathbf{1} ; f(P)=\mathbf{0}$, otherwise. This rule satisfies meet separability and non-imposition but violates upper unanimity.

Rule 3. The lattice polynomial, or majority rule: Let $G \equiv\left\{S \subseteq N:|S|>\frac{N}{2}\right\} \cdot f(P)=$ $\bigvee_{S \in G} \bigwedge_{i \in S} P_{i}$. This rule satisfies upper unanimity and non-imposition but violates meet separability.

This completes the proof.

$\langle\alpha \beta\rangle \vee\langle\alpha \gamma\rangle \geq\langle\beta \gamma\rangle$. This would contradict the assumption that there is no $s \in J \backslash\{p, q\}$ such that $p \vee q \geq s$. So there must be a distinct $\alpha, \beta, \gamma, \delta$ such that $p=\langle\alpha \beta\rangle$ and $q=\langle\gamma \delta\rangle$. It follows then that there must also be elements $r \equiv\langle\alpha \gamma\rangle, s \equiv\langle\beta \gamma\rangle$, and $t \equiv\langle\alpha \delta\rangle$. 


\section{References}

Aigner, M. (1979): Combinatorial Theory, Springer-Verlag.

Barthélemy, J. P. (1988): "Comments on "aggregation of equivalence relations" by P. C.

Fishburn and A. Rubinstein," Journal of Classification, 5, 85-87.

Barthélemy, J.-P., B. Leclerc, And B. Monjardet (1986): "On the use of ordered sets in problems of comparison and consensus of classifications," Journal of Classification, $3,187-224$.

Birkhoff, G. (1973): Lattice Theory, American Mathematical Society, third ed.

Blyth, T. And M. Janowitz (1972): Residuation Theory, Pergamon Press.

Davey, B. A. And H. A. Priestley (2002): Introduction to Lattices and Order, Cambridge University Press, second ed.

Day, W. H. AND F. R. MCMorRIs (2003): Axiomatic Consensus Theory in Group Choice and Biomathematics, Society for Industrial and Applied Mathematics.

Dimitrov, D., T. Marchant, And D. Mishra (2009): "Separability and Aggregation of Equivalence Relations," Working Paper.

Fishburn, P. C. And A. Rubinstein (1986): "Aggregation of Equivalence Relations," Journal of Classification, 3, 61-65.

Grätzer, G. (2003): General Lattice Theory, Birkhäuser Verlag, second ed.

Holmstrom, B. And R. B. Myerson (1983): "Efficient and Durable Decision Rules with Incomplete Information," Econometrica, 51, 1799-1819. 
LeClerc, B. (1984): "Efficient and Binary Consensus Functions on Transitively Valued Relations," Mathematical Social Sciences, 8, 45-61.

Miller, A. D. (2008): "Group Identification," Games and Economic Behavior, 63, 188202.

Mirkin, B. G. (1975): "On the Problem of Reconciling Partitions," in Quantitative Sociology: International Perspectives on Mathematical and Statistical Modeling, ed. by H. M. Blalock, Academic Press, chap. 15, 441-449.

Nehring, K. (2006): "Oligarchies in Judgment Aggregation," Working Paper.

Ore, Ø. (1942): "Theory of Equivalence Relations," Duke Math. Journal, 9, 573-627.

Roman, S. (2008): Lattices and Ordered Sets, Springer.

SzAsz, G. (1964): Introduction to Lattice Theory, Academic Press.

VAnnuCCI, S. (2008): "The Libertarian Identification Rule in Finite Atomistic Lattices," Working Paper.

Wilson, R. (1978): "Information, Efficiency, and the Core of an Economy," Econometrica, $56,807-816$. 